\title{
Editorial
}

\section{Modeling and Control of Complex Networked Systems}

\author{
Guoqiang Hu, ${ }^{1}$ Wenwu Yu, ${ }^{2}$ Guanghui Wen, ${ }^{2}$ Housheng Su, ${ }^{3}$ and Ying $\operatorname{Tan}^{4}$ \\ ${ }^{1}$ School of Electrical and Electronic Engineering, Nanyang Technological University, Singapore \\ ${ }^{2}$ Research Center for Complex Systems and Network Sciences, Department of Mathematics, Southeast University, Nanjing, China \\ ${ }^{3}$ School of Automation, Huazhong University of Science and Technology, Wuhan, China \\ ${ }^{4}$ Department of Electrical and Electronic Engineering, The University of Melbourne Parkville, VIC, Australia
}

Correspondence should be addressed to Guoqiang Hu; gqhu@ntu.edu.sg

Received 8 July 2014; Accepted 8 July 2014; Published 21 July 2014

Copyright (C) 2014 Guoqiang Hu et al. This is an open access article distributed under the Creative Commons Attribution License, which permits unrestricted use, distribution, and reproduction in any medium, provided the original work is properly cited.

Complex networked systems refer to networks of interacting agents, which have been actively studied due to their fundamental scientific, technological, financial, and societal significance. The commonly studied complex networks include but are not limited to social networks, communication networks, sensor networks, actuator networks, economic networks, transportation networks, biological networks, power networks, and autonomous robot networks.

This special issue focuses on modeling and control of complex networked systems. It tries to understand not only the complex networked systems' characteristics but also the design of control strategies to achieve desired collective behaviors.

Call for papers has been carefully prepared by the guest editors and posted on the journal's web page, which has received much attention from researchers in different scientific communities. We have received 31 papers in this research fields. All manuscripts submitted to this special issue went through a thorough peer-refereeing process. Based on the reviewers' reports, 15 original research articles are finally accepted. The contents of this special issue contain modeling, analysis and control of complex networks, collective behaviors in multiagent systems, and analysis and synthesis of complex networked systems. The contents are summarized as follows.

(1) Modeling, Analysis, and Control of Complex Networks. In "A dynamic microblog network and information dissemination in “@” mode" by M. Tang et al., a dynamic microblog model is proposed based on the users' behaviors. "Reorganizing complex network to improve large-scale multiagent teamwork" by $\mathrm{Y}$. Xu et al. studies some factors that affect team performance and proposes an integrated network adjustment algorithm to reorganize the network to expedite the team performance. "A comparison of online social networks and real-life social networks: a study of Sina Microblogging," by D. Zhang and G. Guo, explores the structural characteristics of Sina Microblogging and reveals the similarities and differences between online social networks and real-life social networks. "Local community detection in complex networks based on maximum cliques extension" by M. Fanrong et al. focuses on detecting local community structure in complex networks. "Matthew effect of the random drift on the evolution of cooperation," by C. Liu and R. Li, studies the effect of random drift on evolutionary Prisoner's dilemma on regular lattices. Synchronizability of a class of local-world dynamical networks is studied in "Synchronization in a novel localworld dynamical network model" by J. Tang and P. Liu. Synchronization problems are considered in "Synchronization control of time-varying complex dynamic network with nonidentical nodes and coupling time-delay" by Y. Zhang et al. and "Adaptive asymptotical synchronization for stochastic complex networks with time-delay and Markovian switching" by X. Jiang et al. These two articles consider the synchronization problem for a class of time-varying complex dynamic network and stochastic complex dynamic networks, respectively. "Pinning Lur'e complex networks via output feedback control," by F. Liu et al., investigates the pinning synchronization in a 
network of Lur'e dynamical systems based on distributed output feedback control without using the full state information of network nodes.

(2) Collective Behaviors in Multiagent Systems. "Distributed cooperative current-sharing control of parallel chargers using feedback linearization," by J. Liu et al., proposes a distributed current-sharing scheme to address the output current imbalance problem for the parallel chargers in the energy storage type light rail vehicle system. In "Formation control of multirobot based on I/O feedback linearization and potential function" by J. Dong el al., a formation control method of multirobot system based on potential function is proposed. A new control law is designed by choosing a proper potential function and employing Lyapunov stability theory.

(3) Analysis and Synthesis of Complex Networked Systems. "Finite-time fault detection for large-scale networked systems with randomly occurring nonlinearity and fault," by Y. Zhang et al., investigates the finite-time fault detection problem for a class of nonlinear quantized large-scale networked systems. Moving target positioning based on a distributed camera network is studied by L. Zhao et al. in "Moving target positioning based on a distributed camera network." "Dynamic request routing for online video-on-demand service: a Markov Decision Process approach," by J. Wan et al., investigates the request routing problem in the CDN-based video-on-demand system in which Markov decision process (MDP) and boundedparameter MDP (BMDP) are used in the formulation of the system. "Unified finite horizon Ho fusion filtering for networked dynamical system," by C. Wen et al., addresses the Ho fusion filtering problem for networked dynamical systems under different measurement arrival scenes in a unified manner.

Note that the selected topics and papers are not a comprehensive representation of the area of the special issue. It is also worth noting that the published papers in this special issue do provide some recent advances in the field of complex networked systems, which could benefit the current research more or less.

\section{Acknowledgments}

We would like to thank the authors for their contributions. We also thank anonymous reviewers for their time and help in assessing the manuscripts. Our special thanks go to the editorial board members of this journal owing to their great support and help for this special issue.

$$
\begin{array}{r}
\text { Guoqiang } \mathrm{Hu} \\
\text { Wenwu Yu } \\
\text { Guanghui Wen } \\
\text { Housheng Su } \\
\text { Ying Tan }
\end{array}
$$




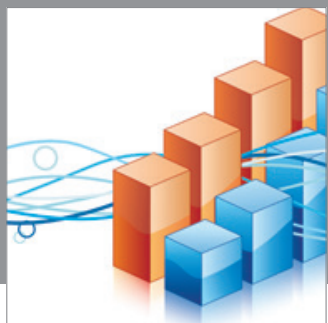

Advances in

Operations Research

mansans

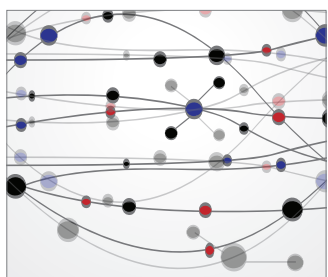

The Scientific World Journal
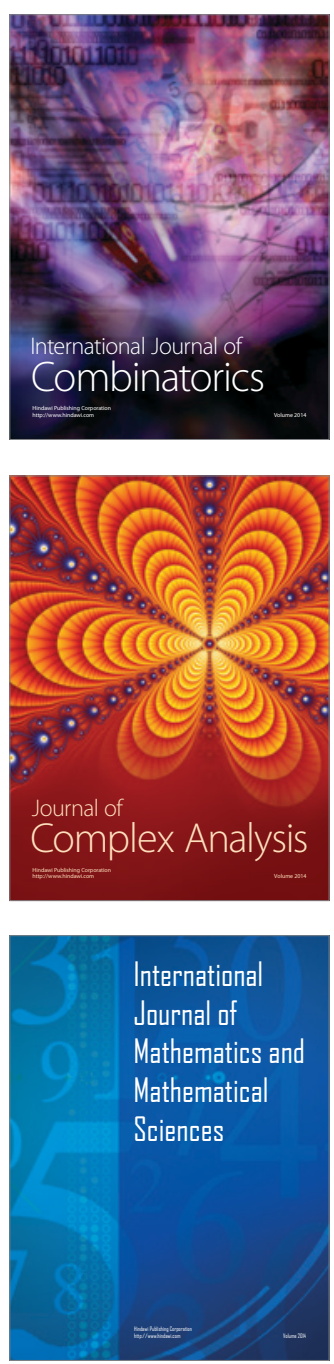
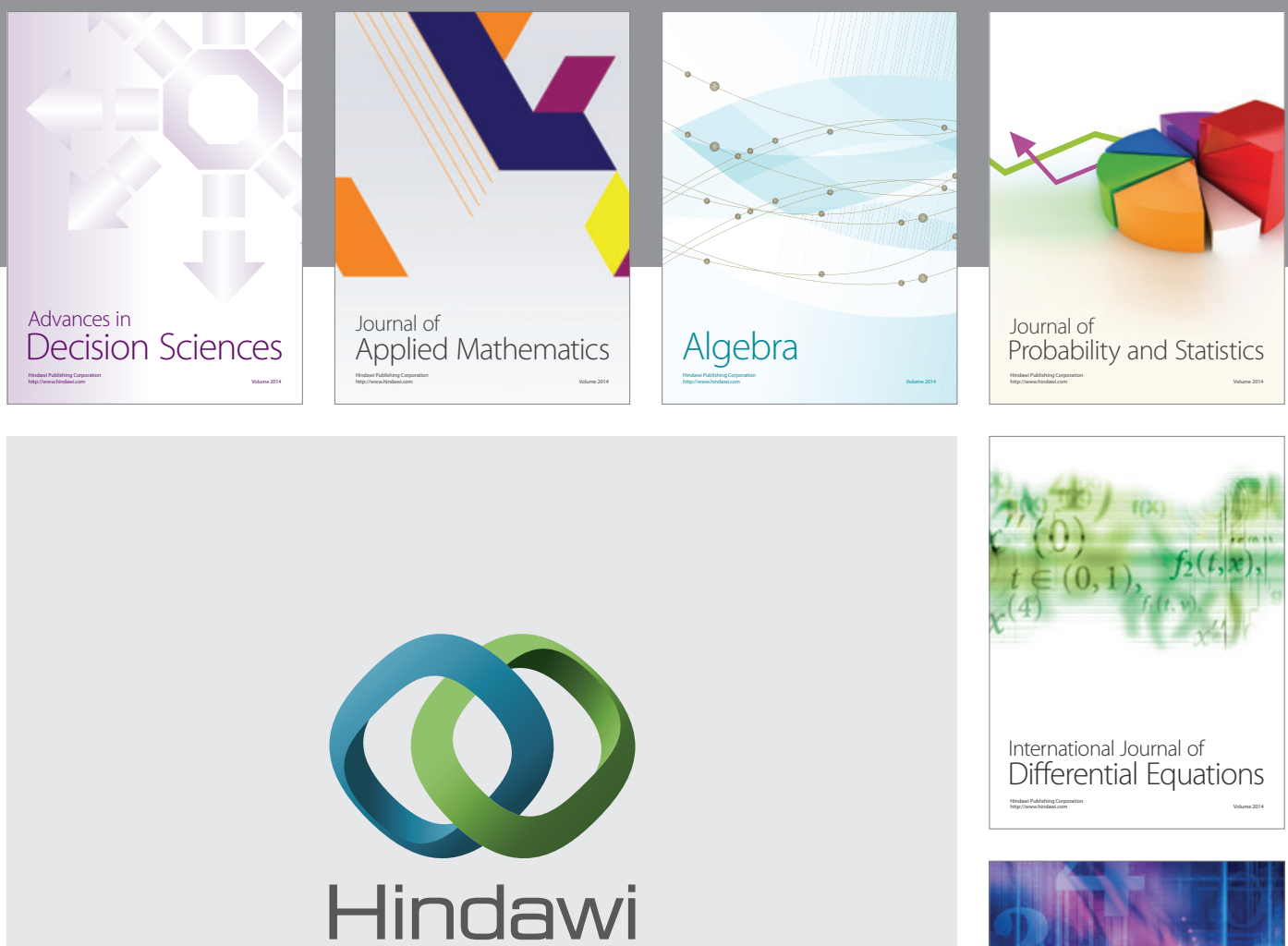

Submit your manuscripts at http://www.hindawi.com
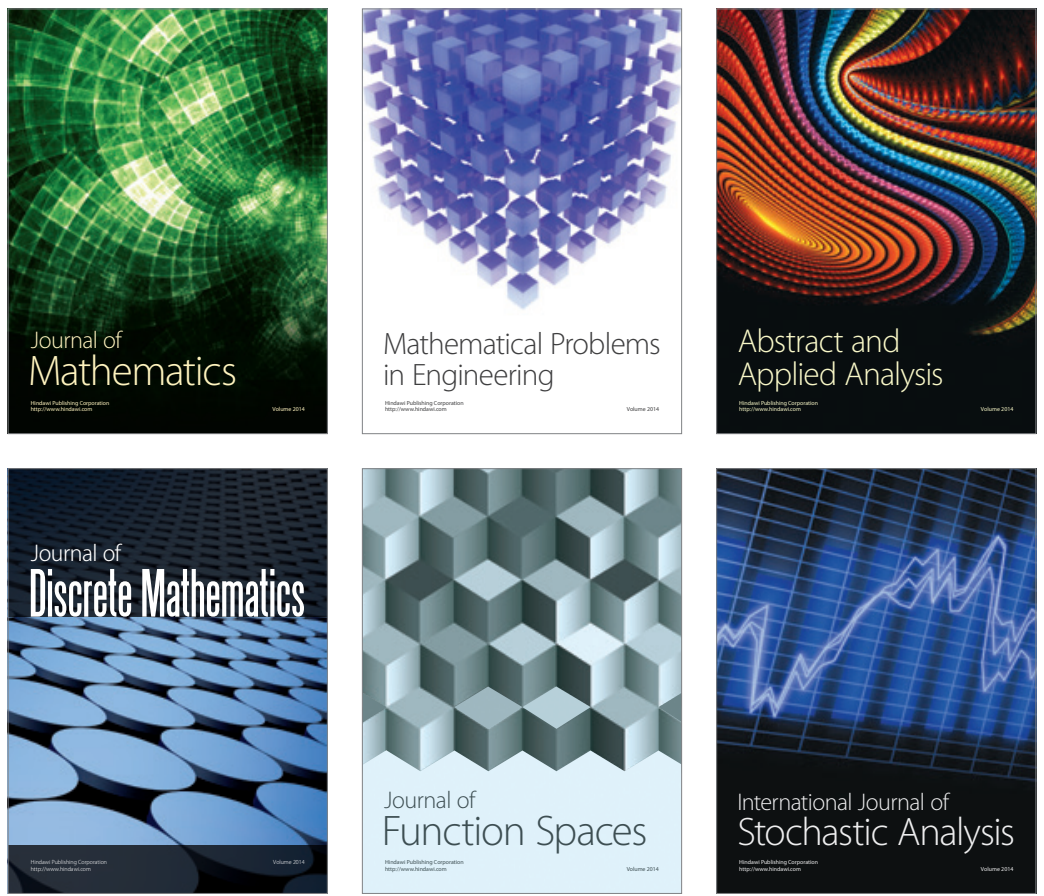

Journal of

Function Spaces

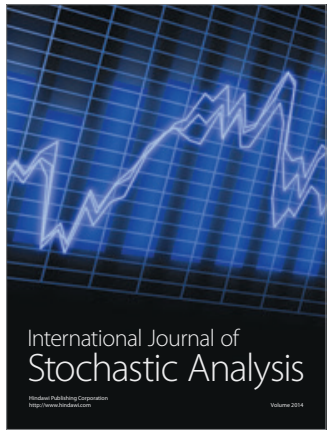

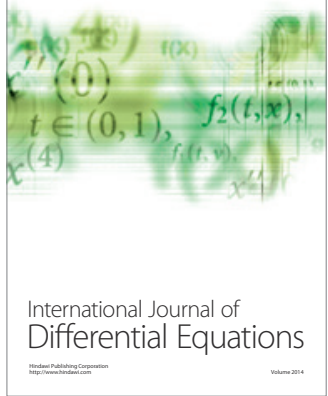
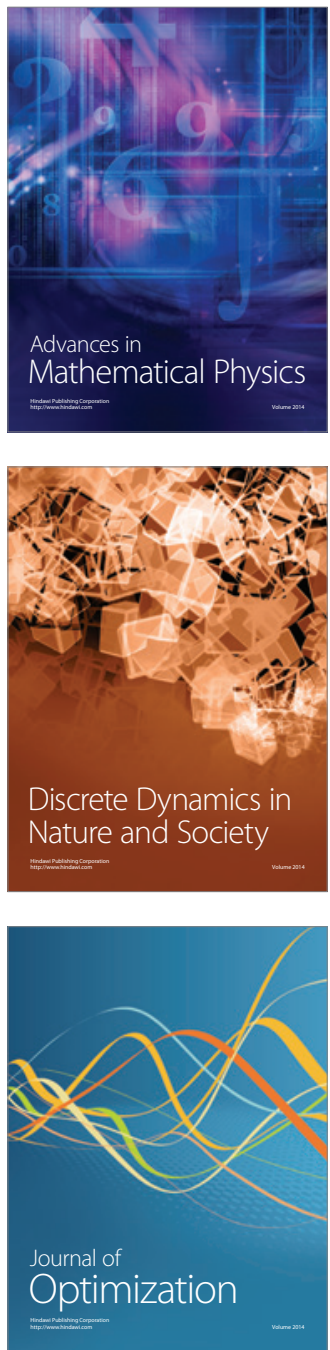